\title{
Science Foundations in cancer research: scope and perspectives
}

When we reflect on the role of Foundations within the context of current healthcare, we need to include the possibility of new forms of management of the complex issues involved. Strictly speaking, in Spain Foundations facilitate options of management types that are of a private or public nature, depending on the source of the sponsorship.

Among the public sources, those that are of note are the Health Foundations set-up under the protection of Statute 15/1997 (25th April) on the Financing of New Forms of Management of the National Health Service, and under Article 111 of Statute 50/1998 (30th December) of Fiscal and Administrative Measures in the Social Regulation of Public Health Foundations.

The other sources are the Foundations promoted by private initiative, which interconnect between the philosophy and the principles relating to the role that civil communities play in generating research and the development of different activities.

Science, in its biomedical aspect, is of great importance since its knowledge directly impacts on the wellbeing of the population. From this perspective, agreements reflect the public authority roles and the significant alternatives that the civil community offers, via Foundations, in generating research and disseminating knowledge.

It is evident that public authorities clearly assume their responsibilities in this field. However, although public authorities have provided, over these past few years, significant budgetary advances via a series of initiatives such as the creation of the Ministry of Science and Technology, there is a wide area of intervention in which the civil community is called-upon to play a central role.

Our constitutional framework embodies a perspective that conceives the citizen as the center of the politicolegal system, and not merely as its recipient. In this sense, the public sphere and the civil community appear related via a social theory that induces, as a modern characteristic, the growing involvement in, and leadership of, the community in the public sphere. This relationship is defined as, amongst other aspects, the recognition of the rights of Foundations.

A Foundation is an instrument serving a general objective according to its capability, sharing the public sphere due to its character, but with a private-legal structure that provides it with a greater flexibility in relation to the specific orientation of its operational remit.
Following-on from this formula, the civil community has a significant opportunity in driving or promoting worthy initiatives, of receiving the recognition and assistance from the public authorities who are aware of the enormous labour that is required in the field of training and scientific research, and to establish identity of all those who are prepared to invest their time and strengths in it.

From another point of view, the organisational structure of a Foundation facilitates those citizens involved in aspects of common interest to put back into society some of the benefits taken out in the various sections and activities, within the framework of principles of social responsibility.

The collaboration that is given to Spanish biomedical research by private entities, especially the pharmaceutical industry, whether directly or through Foundations, has contributed to R\&D situation in Spain, which has improved notoriously in these past few years. The number of researchers has increased substantially and has improved our scientific production in which we have gone from being mere consumers of advances developed in other countries to being producers of scientific knowledge.

Those of us from the private health sector that believe in the Foundation as a formula of collaboration, believe that health is an indispensable service in settingup schemes within a modern structure and whose existence, guaranteed by public administrations in fulfilling constitutional requirements, which need to be favored by all for the benefit of all.

A health network is complex and in it is present a wide range of participants. Firstly, there are the citizens who, by reason of their taxes, have the right to participate and to receive the best support from the available health-care resources. Secondly, there are the public institutions that guaranty the resources, their procurement and co-ordination while ensuring that they reach the citizen under the best conditions and criteria of efficiency. Thirdly, there are the manufacturers and providers of the very diverse services who ensure their accessibility under conditions of maximum quality.

This last group includes the pharmaceutical industry, with an important development and a deeply rooted tradition in Spain, that in the general setting within which it operates, is experiencing profound changes that shall allow it to respond to the expectations of society. 
The first mission of the pharmaceutical industry is to produce drugs that contribute to the maintenance, or to the recovery, of the health of citizens within the limits of current knowledge. This requires that the sector participate in research, the generation of knowledge and its translation into the production of treatments for diseases. In other words, industry needs permanently to encourage innovation and to improve the therapeutic arsenal.

But there are other ways, apart from the generation of wealth and employment, in which the industry can collaborate in this social benefit program. These include assistance in biomedical research, the dissemination of knowledge, the creation of forums of opinion and interchange of ideas, health-care education, disease prevention, etc.

From this reasoning, and with the conviction of the need to contribute through these activities to the better development and advancement of our health, the Lilly Foundation has proposed to channel and favor initiatives for social and health-care collaboration in Spain; the general objectives being to contribute to the development and improvement of the health of citizens by prompting programs of research, education, dissemination, prevention, consultation, technical assistance and the promotion of projects.

New advances in genomic research and the new technologies make possible a flowering of new information and scientific knowledge that are essential in the fight against cancer and other diseases. However, and despite of the visible improvements in the management and treatment of these diseases, the transfer of the new knowledge into the clinical situation has not been at a desirable speed.

In the case of cancer, it is gratifying to see advances in basic knowledge proceed so rapidly. The deciphering of the human genome is opening-up knowledge of details of the molecular environment of the disease and, more importantly, identifying new experimental designs in investigating the human tumors-of-interest and to propose new methods of treatment. However, the rate of progress in clinical oncology is very slow. A prestigious clinical oncologist recently described his frustration by highlighting that the "achievements in the treatment of cancer over the last decade have been more in the advances in surgical techniques and the treatment of adverse effects of adjuvant treatment than in the real capacity to kill the tumor cells". As a result, the achievements in cancer treatment have re- ached a crossroad in that cytotoxic chemotherapy does not appear to provide, in the immediate future, much improved strengthening of the therapeutic arsenal. This encourages those interested, to return to the search for a new generation of pharmacological agents that would impact directly on the genes implicated in the development of cancer.

For this reason, and given that cancer is currently the second biggest cause of death in the western world, it is not surprising that the majority of the pharmaceutical companies have included oncology in their pursuit of the discovery of new drugs. The outcome, most probably, would be the development and application of these new treatments, especially those indicated for solid tumours that will require multidisciplinary approaches. These include molecular pathology and pharmaco-genomic techniques for more appropriate treatment of "each patient and his tumour" and to assist in the decision-making process of the clinical oncologist.

Now, more than ever, there is an urgent necessity to combine the strengths of basic scientists with those of clinical oncologists with their respective knowledge and experience. From this conviction, the Lilly Foundation proposes to promote the interchange of ideas and knowledge between the pre-clinical and clinical communities, not only in cancer but also in all the areas of biomedical research in which there are challenges to confront.

We need to be conscious, as a country, of the absolute necessity of participating with redoubled efforts in the research and development of solutions, the implication of which for the National Health System, is evident.

Our scientific community draws on a history of poor investment and communal estrangement of science. It would require considerable effort to recover the time lost and to sustain leading projects as the Centro $\mathrm{Na}$ cional de Investigaciones Oncológicas Carlos III: CNIO [National Centre for Cancer Research Carlos III]. New initiatives, such as the recent Plan Integral Contra el Cáncer [Integrated Plan Against Cancer] with a national ambit and supported by the Ministry of Health, identify important targets or milestones that require new investment, both public as well as private.

José Antonio Gutiérrez Fuentes Director Fundación Lilly. Madrid. 